\title{
Tensor Velocity Imaging with Motion Correction
}

Jørgensen, Lasse Thurmann; Schou, Mikkel; Stuart, Matthias Bo; Jensen, Jørgen Arendt

Published in:

2020 IEEE International Ultrasonics Symposium

Link to article, DOI:

10.1109/IUS46767.2020.9251661

Publication date:

2020

Document Version

Peer reviewed version

Link back to DTU Orbit

Citation (APA):

Jørgensen, L. T., Schou, M., Stuart, M. B., \& Jensen, J. A. (2020). Tensor Velocity Imaging with Motion Correction. In 2020 IEEE International Ultrasonics Symposium IEEE.

https://doi.org/10.1109/IUS46767.2020.9251661

\section{General rights}

Copyright and moral rights for the publications made accessible in the public portal are retained by the authors and/or other copyright owners and it is a condition of accessing publications that users recognise and abide by the legal requirements associated with these rights.

- Users may download and print one copy of any publication from the public portal for the purpose of private study or research

- You may not further distribute the material or use it for any profit-making activity or commercial gain

- You may freely distribute the URL identifying the publication in the public portal

If you believe that this document breaches copyright please contact us providing details, and we will remove access to the work immediately and investigate your claim. 


\title{
Tensor Velocity Imaging with Motion Correction
}

\author{
Lasse Thurmann Jørgensen, Mikkel Schou, Matthias Bo Stuart, Jørgen Arendt Jensen
}

Center for Fast Ultrasound Imaging, Department of Health Technology, Technical University of Denmark

\begin{abstract}
Volumetric synthetic aperture imaging allows for tensor velocities to be estimated in the entire image volume at high frame rates. However, the approach assumes that scatterers are stationary across the low-resolution images. If not, motion effects can cause a severe decrease in the accuracy of the velocity estimates. This paper presents a motion compensation procedure that reduces motion effects in synthetic aperture tensor velocity estimates acquired with a row-column addressed probe. The procedure reduces the motion effects by translating the image coordinates of the low-resolution images with the velocity field. The velocity field is estimated using a transverse oscillation crosscorrelation estimator, and the flow measurements are acquired using a $3 \mathrm{MHz}$ row-column addressed probe and the research scanner SARUS. For a peak velocity of $25 \mathrm{~cm} / \mathrm{s}$, a pulse repetition frequency of $2 \mathrm{kHz}$, and a beam-to-flow of 60 degrees, the proposed motion compensation procedure was able to reduce the relative bias from $-\mathbf{2 7 . 0 \%}$ to $\mathbf{- 8 . 3 \%}$ without impacting the relative mean standard deviation of $8.8 \% \pm 0.1 \%$.
\end{abstract}

Index Terms-Motion correction, Tensor velocity imaging, Row column addressed probes, volumetric imaging.

\section{INTRODUCTION}

Tensor velocity imaging (TVI) shows a 3-D velocity vector at high frame rates at each point in the volume. TVI has only recently been achievable with advances in 2-D probe technology and volumetric imaging techniques. In [1] TVI was achieved using a $32 \times 32$ fully addressed 2-D array probe. Here five lines were beamformed in parallel to acquire 3-D velocity estimations along a flow line using the transverse oscillation (TO) approach, developed by Jensen and Munk [2], [3]. A similar line-by-line TVI approach is presented in [4] but by utilizing a $62+62$ row-column addressed array (RCA) probe [5]. The RCA array has the advantage of only requiring $N+N$ channels to cover the same surface area of an $N \times N$ fully addressed array. This is because the RCA array addresses the 2-D matrix array elements by rows and columns instead of having one-to-one element channel connectivity. However, the trade-off is that the RCA does not have the same steering flexibility of the fully addressed array. The RCA probe architecture makes it less challenging to increase the number of elements along the array's side-length, and it is thus more feasible to maintain a low F-number at large imaging depth. In [6], cardiac TVI was achieved using a modified GE Vivid E95 ultrasound scanner (GE Vingmed, Horten, Norway) where sub-aperture beamforming was implemented to reduce the number of channels of the 2-D probe. Here tensor velocity was estimated using 3-D block-matching, and full volumetric coverage of the left ventricle was achieved using ECG-gating.

These works [1], [4], [6] presents TVI systems that acquire a limited amount of flow data within a specific time frame. However, a slow volumetric coverage limits the applications of TVI, and it is, therefore, worth seeking improvements to the rate of the volumetric coverage, i.e., the volume rate. Volumetric synthetic aperture (SA) imaging techniques provide a solution to the volume rate demand and can acquire continuous flow data throughout the entire image volume simultaneously. The SA emission sequence resolves the image volume by isonifying the volume with defocused emissions from different angles. Each emission yields a low-resolution volume (LRV), and combining LRVs from different emission sources creates dynamic transmit focusing because the LRVs sum coherently at the scatterers' positions. Combining the LRVs from the entire emission sequence yields a high-resolution volume (HRV), which is dynamically focused in both transmit and receive.

SA TVI has been demonstrated with a fully addressed probe in [7] and with an RCA probe in [8]. The results presented in [8] shows that the method works for transverse motions, but that accuracy decreases when axial motion is present. The reduction in accuracy is hypothesized to be caused by motion effects. The motion effects occur because moving scatterers cause phase misalignment between LRVs. Other researchers have studied the effects of motion [9]-[12] in SA imaging systems and lateral motion is reported to broaden the pointspread function (PSF) and axial motion have been found to cause a spatial shift in the PSF. The latter is far more severe as it changes the moving object's geometry and thus reduces the imaging accuracy. The motion effects can be reduced by correcting the LRVs' inter-frame motion before summation [10], [12]. Here the velocity at each image-point is estimated and used to deform the low-resolution images such that there is a reduction in motion between them.

This work investigates the performance of SA TVI when correcting for 3-D motion using tensor velocity estimates. The proposed motion compensation procedure's performance is evaluated on a flow-rig measurement acquired with an RCA array at a beam-to-flow angle of $60^{\circ}$. At this angle, axial motion is present, and the flow profiles with and without motion correction are compared.

\section{MOTION COMPENSATION}

The motion compensation procedure aims to negate the motion between the LRVs constituting the HRV. This is achieved by translating the image-points of the LRV with the estimated flow field. This 3-D translation of the image point, $\Delta \vec{p}=(\Delta x, \Delta y, \Delta z)$, is given by

$$
\Delta \vec{p}=(j-(M+1) / 2) \frac{\vec{v}}{f_{p r f}},
$$


where $f_{\text {prf }}$ denotes the pulse repetition frequency, $\vec{v}=$ $\left(v_{x}, v_{y}, v_{z}\right)$ is the local velocity vector and $j \in[1, \ldots, M]$ denotes of the index of the $M$ LRVs. Note that the velocity is assumed constant throughout the summation period. This is because the velocity is not updated per emission, but per $2 M$ emissions, which is the minimum amount necessary to estimate the three velocity components with the proposed TVI approach (see Section IV-A). Note also that the LRV image points' translation is smallest in the LRVs half-way through the emission sequence. This is to ensure that the maximum translation distance is as small as possible.

Motion-corrected velocity estimation is inherently a recursive problem, as it requires velocity estimates to obtain new estimates with reduced motion effects. The proposed motion compensation algorithm can be expressed by substituting the velocity term in (1) by the average velocity estimate over iterations:

$$
\bar{v}_{k}=\frac{1}{k-1} \sum_{i=1}^{k-1} \vec{v}_{i} \quad \text { for } k=2,3 \ldots
$$

where $\vec{v}_{i}$ is denotes the $i$ th iteration of the velocity estimate. Note that $\Delta \vec{p}=0$ in the base case, where the first iterations of the velocity estimations have not been performed.

\section{TENSOR VELOCITY ESTIMATOR}

The tensor velocity is estimated using the cross-correlation directional TO estimator presented in [8]. The approach estimates the three velocity components by beamforming along each direction and determining the motion through crosscorrelation. The velocity along the axial lines can be estimated directly because the emitted field is inherently sensitive to axial motion due to the pulse's axial frequency component. This is, however, not the case when estimating the velocity in the other directions, and the TO method relies on generating transverse oscillations to increase the sensitivity to transverse motion. This is achieved by applying double peak apodization in receive, which, following the Fraunhofer diffraction equation, generates a field with a transverse wavelength of [2]

$$
\lambda_{T O}=2 \lambda \frac{z}{D}
$$

Here $\lambda$ is the wavelength of the emitted pulse, and $D$ is the distance between the two peaks of the receive apodization. It is also necessary to filter out the axial oscillations in the field to estimate the transverse velocity as they can cause de-correlation. The filtering was performed following the procedure presented in [13], where the axial oscillation is reduced by combining outputs of the Hilbert transform along the axial and transverse direction.

Because the double peak apodization only is applied in receive, separate emissions are needed to estimate velocities along the $x$ - and $y$-directions. With the implemented method, the transverse oscillation is only generated along the receiving axis, and the orientation of this axis depends on whether it is the row or column aperture that receives the backscattered signal. Therefore, to obtain both the $x$ and $y$ velocity components, it is necessary to interchange the transmitting and receiving aperture. It should be mentioned that it is theoretically possible to induce a TO along both directions without this interchanging the apertures. This can be achieved by applying double peak apodization to the synthetic transmit aperture. The effectiveness of this approach has yet to be investigated and is a topic for future research.

The velocity along the beamformed lines are estimated using the normalized cross-correlation, which is defined by:

$$
R_{12}(m)=\frac{\sum_{n=-\infty}^{\infty} r_{1}^{*}(n) r_{2}(n+m)}{\sigma_{1} \sigma_{2}}
$$

where $r_{1}^{*}(n)$ is the complex conjugate of $r_{1}(n)$, and $r_{1}(n)$ and $r_{2}(n)$ denote two consecutive signals in $\mathbb{C}^{N}$ with the standard deviation of $\sigma_{1}$ and $\sigma_{2}$. The motion between the two signals is then determined by the peak of the real part of $R_{12}(m)$. The peak is determined from the real-part since it has been found to yield a more precise result [13]. The lag of the peak is obtained by the argument of the maximum of the crosscorrelation function:

$$
m_{p}=\underset{m}{\operatorname{argmax}}\left(\operatorname{Re}\left(R_{12}(m)\right)\right) .
$$

The lag is then adjusted such that it locates the maximum of a second-order polynomial fitted to the peak of $R_{12}(m)$ and its neighboring points:

$$
m_{p q}=m_{p}-\frac{R_{12}\left(m_{p}+1\right)-R_{12}\left(m_{p}-1\right)}{R_{12}\left(m_{p}+1\right)-2 R_{12}\left(m_{p}\right)+R_{12}\left(m_{p}-1\right)} .
$$

The adjustment allows for continuous lag values and thus reduces the need for a high sampling frequency. The velocity is obtained from the adjusted lag by the following conversion:

$$
v=m_{p q} \frac{c f_{p r f, e f f}}{2 f_{s}},
$$

where $c$ is speed of sound, $f_{s}$ is the sampling frequency and $1 / f_{p r f, e f f}$ is the effective pulse repetition period, which is the duration between $r_{1}(n)$ and $r_{2}(n)$.

\section{Methodology}

\section{A. Emission sequence}

The emission sequence consists of eight defocused emissions linearly spaced by $2.2 \lambda$. The emission sequence alternates between being emitted by the row and column aperture after obtaining one HRV pair, i.e., 16 emissions. The emission sequence from both the row and column apertures is visualized in Fig. 1. The back-scattered signal is measured by all receiving elements in parallel and receive apodization and focusing is applied off-line. The 16 emissions used to acquire one HRV pair are emitted using an interleaved emission sequence, where each emission is repeated twice before changing the focal line position. The odd and even emissions are then separately used to form the two HRVs, which, because of the interleaving, are separated in time by one pulse repetition period, $1 / f_{p r f}$. The cross-correlation functions are averaged over $10 \mathrm{HRV}$ pairs and updates each time one pair is acquired. This means that the proposed sequence yields new tensor velocity estimates for every 16 emissions. 
TABLE I

PARAMETER FOR TVI MEASUREMENTS

\begin{tabular}{lccc}
\hline Parameter & Value & Unit & Variable \\
\hline $\begin{array}{l}\text { No. elements } \\
\text { Center frequency }\end{array}$ & $62+62$ & & \\
Excitation pulse & 2 cycled sin. & $\mathrm{MHz}$ & $f_{0}$ \\
$\begin{array}{l}\text { Element pitch } \\
\text { Transmit apodization }\end{array}$ & 0.27 & $\mathrm{~mm}$ & \\
von Hann & & \\
$\begin{array}{l}\text { No. active elements } \\
\text { F-number }\end{array}$ & 32 & & \\
$\begin{array}{l}\text { Pulse repetition frequency } \\
\text { Sampling frequency }\end{array}$ & -1 & & \\
No. emissions & 17.5 & $\mathrm{kHz}$ & $f_{p r f}$ \\
$\begin{array}{l}\text { Beam-to-flow angle } \\
\text { flow-rotation angle }\end{array}$ & 8 & & \\
Peak velocity & 60 & Degrees $\left(^{\circ}\right)$ & $\alpha$ \\
\hline & $10 \& 25$ & Degrees $\left(^{\circ}\right)$ & $\beta$ \\
\end{tabular}

\section{B. Flow-rig measurements}

The flow-rig measurements were performed using a $3 \mathrm{MHz}$ $62+62$ RCA probe coupled to the research scanner SARUS [14]. The probe was placed in a transducer fixture, with two rotation angles, which allows the operator to change both the beam-to-flow angle, $\alpha$, and the flow-rotation angle, $\beta$. During the measurements, $\alpha$ was set to $60^{\circ}$, and $\beta$ was set to $45^{\circ}$. Note that at this rotation, the flow contains velocity components in all three directions.

At the measurement site, a straight rubber vessel with a 12 $\mathrm{mm}$ diameter carries parabolic flow, and the volume rate is monitored with a Danfoss Magnetic flow meter MAG1100. The measurement parameters are summarized in table I, and the setup is illustrated in Fig. 1.

\section{RESULT AND DISCUSSION}

The measurements were performed with a pulse repetition frequency of $2 \mathrm{kHz}$, and the 3-D velocities were estimated along the central axial line. When correcting for the motion, three iterations of the motion compensation algorithm was used (see Section II). The estimator's performance was evaluated from the relative mean bias, $B$, and standard deviation, $\bar{\sigma}$ of the velocity magnitude profile. The performance measures were calculated from 200 realizations of the magnitude profile, and only the central $90 \%$ of the profile was used in the calculations to avoid vessel border effects. The results are shown in Fig 2. The images in the figure's left column show the velocity profiles without motion compensation, and the images in the right column show the profiles with motion compensation. At a peak velocity of $10 \mathrm{~cm} / \mathrm{s}$, the motion compensation manages to reduce $B$ by $6.4 \%$ from $-6.5 \%$ to $0.1 \%$. At a peak velocity of $25 \mathrm{~cm} / \mathrm{s}$, the motion compensation manages to reduce $B$ by $18.7 \%$ from $-27.0 \%$ to $-8.3 \%$. Also, the profiles obtained after motion compensation are qualitatively more similar to the theoretical profiles. The motion compensation procedure has little to no impact on $\bar{\sigma}$; at a peak velocity of $10 \mathrm{~cm} / \mathrm{s}$, the motion compensation reduces $\bar{\sigma}$ by $0.5 \%$ and at a peak velocity of $25 \mathrm{~cm} / \mathrm{s}$, the motion compensation causes $\bar{\sigma}$ to increase by $0.1 \%$. Thus, the improvement in bias does not seem to come at the cost of an increased standard deviation.

\section{CONCLUSION}

The proposed 3-D motion compensation algorithm reduced SA TVI estimates' bias in measurements acquired with a $3 \mathrm{MHz} 62+62$ RCA transducer, a beam-to-flow angle of $60^{\circ}$ and a pulse repetition frequency of $2 \mathrm{kHz}$. The largest reduction in bias $(18.7 \%$ ) was obtained in measurement where the flow's peak velocity was $25 \mathrm{~cm} / \mathrm{s}$. The substantial increase in accuracy was found not to come at the cost of precision, and the measurements showed a negligible difference in the standard deviation of $0.1 \%$. The accuracy increase shows that the proposed motion compensation procedure is a viable solution to the motion effects found in RCA SA imaging systems.

\section{REFERENCES}

[1] M. J. Pihl and J. A. Jensen, "A transverse oscillation approach for estimation of three-dimensional velocity vectors. Part I: Concept and simulation study," IEEE Trans. Ultrason., Ferroelec., Freq. Contr, vol. 61, pp. 1599-1607, 2014.

[2] J. A. Jensen and P. Munk, "A new method for estimation of velocity vectors," IEEE Trans. Ultrason., Ferroelec., Freq. Contr., vol. 45, no. 3, pp. 837-851, 1998.

[3] J. A. Jensen, "A new estimator for vector velocity estimation," IEEE Trans. Ultrason., Ferroelec., Freq. Contr., vol. 48, no. 4, pp. 886-894, 2001.

[4] S. Holbek, T. L. Christiansen, M. B. Stuart, C. Beers, E. V. Thomsen, and J. A. Jensen, "3-D vector flow estimation with row-column addressed arrays," IEEE Trans. Ultrason., Ferroelec., Freq. Contr., vol. 63, no. 11, pp. 1799-1814, 2016.

[5] C. E. Morton and G. R. Lockwood, "Theoretical assessment of a crossed electrode 2-D array for 3-D imaging," in Proc. IEEE Ultrason. Symp., pp. 968-971, 2003.

[6] M. S. Wigen, S. Fadnes, A. Rodriguez-Molares, T. Bjåstad, M. Eriksen, K. H. Stensæth, A. Støylen, and L. Løvstakken, "4-D intracardiac ultrasound vector flow imaging-reasibility and comparison to phasecontrast MRI," IEEE Trans. Med. Imag., vol. 37, pp. 2619-2629, December 2018.

[7] M. Correia, J. Provost, M. Tanter, and M. Pernot, "In-vivo 4D ultrafast vector flow imaging: quantitative assessment of arterial blood flow," in Proc. IEEE Ultrason. Symp., pp. 1-4, 2016.

[8] M. Schou, L. Jørgensen, C. Beers, M. S. Traberg, B. G. Tomov, M. B. Stuart, and J. A. Jensen, "Fast 3-d velocity estimation in 4-d usinga 62 + 62 row-column addressed array," IEEE Trans. Ultrason., Ferroelec., Freq. Contr., vol. -, no. -, pp. -, 2020.

[9] G. E. Trahey and L. F. Nock, "Synthetic receive aperture imaging with phase correction for motion and for tissue inhomogenities - part II: effects of and correction for motion," IEEE Trans. Ultrason., Ferroelec. Freq. Contr., vol. 39, pp. 496-501, 1992.

[10] B. Denarie, T. A. Tangen, I. K. Ekroll, N. Rolim, H. Torp, T. Bjastad, and L. Løvstakken, "Coherent plane wave compounding for very high frame rate ultrasonography of rapidly moving targets," IEEE Trans. Ultrason., Ferroelec., Freq. Contr., vol. 32, no. 7, pp. 1265-1276, 2013.

[11] N. Oddershede and J. A. Jensen, "Effects influencing focusing in synthetic aperture vector flow imaging," IEEE Trans. Ultrason., Ferroelec., Freq. Contr., vol. 54, no. 9, pp. 1811-1825, 2007.

[12] K. L. Gammelmark and J. A. Jensen, "2-D tissue motion compensation of synthetic transmit aperture images," IEEE Trans. Ultrason., Ferroelec., Freq. Contr., vol. 61, pp. 594-610, April 2014.

[13] J. A. Jensen, "Estimation of high velocities in synthetic aperture imaging: II: Experimental investigation," IEEE Trans. Ultrason., Ferroelec., Freq. Contr., vol. 66, no. 6, pp. 1032-1038, 2019.

[14] J. A. Jensen, H. Holten-Lund, R. T. Nilsson, M. Hansen, U. D. Larsen, R. P. Domsten, B. G. Tomov, M. B. Stuart, S. I. Nikolov, M. J. Pihl, Y. Du, J. H. Rasmussen, and M. F. Rasmussen, "SARUS: A synthetic aperture real-time ultrasound system," IEEE Trans. Ultrason., Ferroelec., Freq. Contr., vol. 60, no. 9, pp. 1838-1852, 2013. 

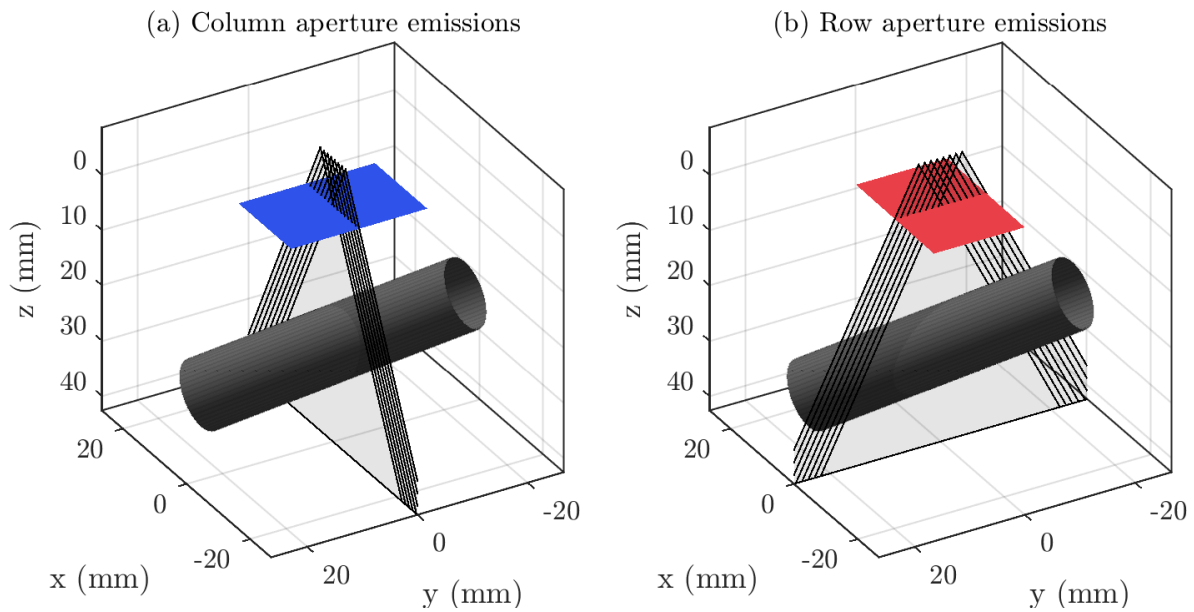

Fig. 1. Geometry of the emission sequence. Blue and red squares show the dimensions of the column- and row apertures. The cylinder indicates a vessel carrying the flow, and the outlined triangles show the emitted fields from eight emission sources.
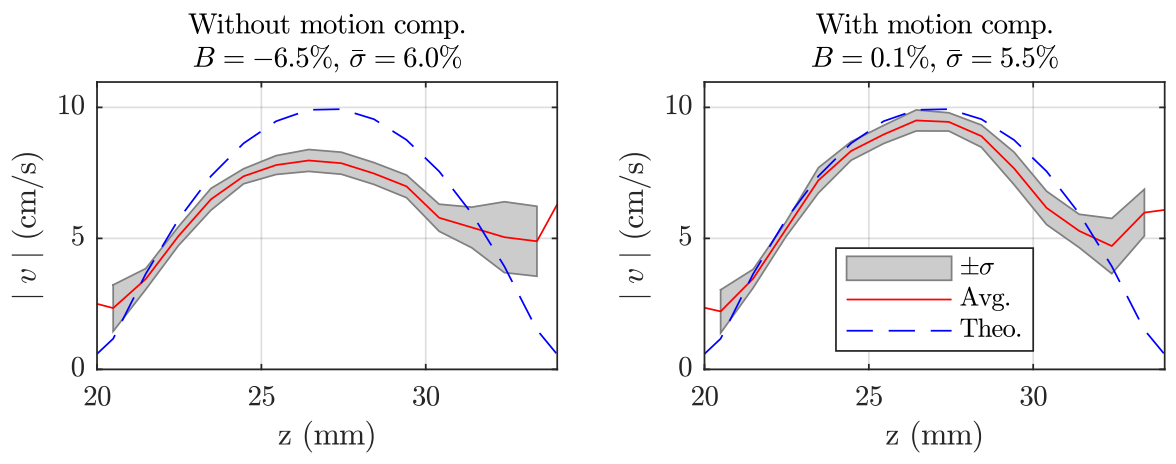

(a) Profiles when peak velocity is $10 \mathrm{~cm} / \mathrm{s}$
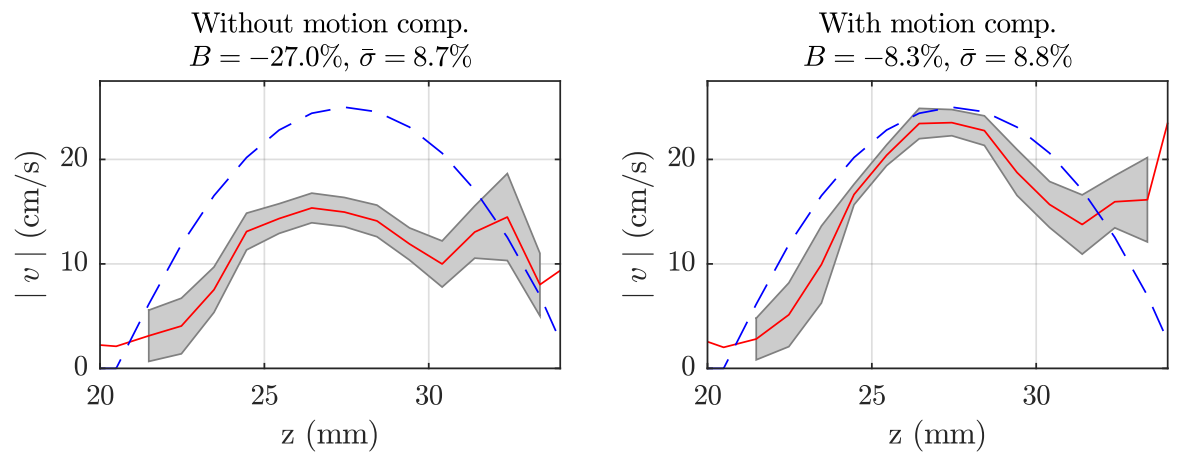

(b) Profiles when peak velocity is $25 \mathrm{~cm} / \mathrm{s}$

Fig. 2. Velocity magnitude profiles from flow-rig measurements. The rightmost profiles are obtained without motion compensation, and leftmost profiles are obtained using motion compensation. (a) shows profiles of flow with a peak velocity of $10 \mathrm{~cm} / \mathrm{s}$, and (b) shows profiles of flow with a peak velocity of 25 $\mathrm{cm} / \mathrm{s}$. The dashed blue line shows the theoretically expected profile. The red line shows the average estimated velocity profile, and the grey area shows the average profile \pm one standard deviation. 